\title{
X-raying the evolution of SN 1987 A
}

\author{
Vinay L. Kashyap ${ }^{1,2}$, David van Dyk ${ }^{3}$, Katy McKeough ${ }^{4}$, Frank \\ Primini $^{1}$, Diab Jerius ${ }^{1}$, Akshay Gowrishankar ${ }^{5}$, Aneta Siemiginowska ${ }^{1}$ \\ and Andreas Zezas ${ }^{1,6}$ \\ ${ }^{1}$ Harvard-Smithsonian Center for Astrophysics, 60 Garden St., Cambridge MA 02138, USA \\ ${ }^{2}$ email: vkashyap@cfa.harvard.edu \\ ${ }^{3}$ Dept. of Statistics, Imperial University, London, UK \\ ${ }^{4}$ Dept. of Statistics, Harvard University, Cambridge MA, USA \\ ${ }^{5}$ Acton-Boxborough Regional High, Acton MA, USA \\ ${ }^{6}$ Dept. of Physics, University of Crete, Heraklion, Greece
}

\begin{abstract}
SN 1987A has been observed with the Chandra X-ray Observatory over the entire course of the mission. We have re-analyzed the archival data by constructing an empirical point spread function and reconstructing high-resolution images using a Bayesian multi-scale image reconstruction algorithm. We are able to resolve structure in the equatorial ring of SN 1987A with unprecedented detail, at scales of $\approx \frac{1}{4}$ arcsec. We describe how the point spread function is constructed, and the reconstruction method, and explore the evolution of the inner ring at different epochs and passbands.
\end{abstract}

Keywords. supernova remnants, supernovae: individual (SN 1987A), circumstellar matter, methods: data analysis, methods: statistical, techniques: image processing, X-rays: general

\section{Introduction}

SN 1987A is the closest supernova of the modern era (at $\approx 50 \mathrm{kpc}$ in the Large Magellanic Cloud), and has served as a touchstone for models of Type II core-collapse supernovae and the subsequent evolution of the ejecta. In the thirty years since the event, it has been studied extensively in multiple wavebands. In the optical, it shows an evolving inner ring and two relatively stationary outer rings (see, e.g., Burrows et al. 1995). The outer rings have been interpreted as light echoes, and the inner ring as due to the blast wave running into dense material. The inner ring was first observed in X-rays with ROSAT (Hasinger et al. 1996). It was first resolved in X-rays with Chandra (Burrows et al. 2000) and its evolution has been continuously monitored since then (see, among many others, Park et al. 2006, Racusin et al. 2009, Helder et al. 2013, Frank et al. 2016). The X-ray intensity had increased continuously, faster since c.2004, but appears to have flattened out and started decreasing since c.2013, especially in the soft and medium bands (see Figure 1). There have been a number of morphological changes, with different parts of the ring brightening and fading. The expansion rate has shown a dichotomy, expanding faster in hard X-rays than in soft X-rays since c.2004, which has been interpreted as due to the blast wave hitting denser material, resulting in strong shocks that are brighter in soft X-rays and move more slowly (Frank et al. 2016).

We have developed new Chandra calibration products and devised image analysis methods that promise to radically improve the inferences that can be drawn from the data. In $\S 2$, we describe how an empirical PSF is made at sub-pixel resolution. In $\S 3$, 


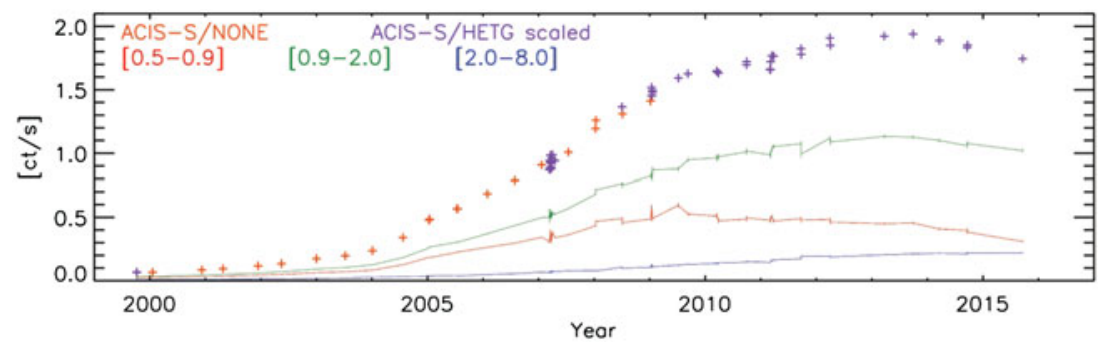

Figure 1. Count rates of SN 1987A in Chandra/ACIS-S. Observations made with bare ACIS-S (red '+') and those made with HETGS+ACIS-S (purple '+') scaled to match the bare ACIS count rates between 2007-2009 are shown, as well as the count rates in the soft (red line), medium (green line), and hard (blue line) passbands.

we describe a Bayesian multi-scale algorithm that we apply to the Chandra images. We then reanalyze Chandra/ACIS data of SN 1987A, and discuss preliminary results in $\S 4$.

\section{Chandra Empirical PSF}

SN 1987A has been observed predominantly with the Chandra/ACIS-S detector, a CCD with pixels $0^{\prime \prime} .49$ arcsec in size, comparable to the size of the point spread function (PSF). We have empirically constructed a PSF based on observations of on-axis, weak, isolated, point sources, and use it to analyze all ACIS-S data. The bare ACIS-S has accumulated $663 \mathrm{ksec}$ of exposure, but most observations after c.2007 have been carried out with the high-energy transmission grating (HETG) in the optical path, accumulating 1.45 Msec of exposure. A few observations have utilized the low-energy transmission grating (LETG) and some have used the HRC-I, a microchannel plate detector; because these observations involve significantly different shapes of the PSF, we ignore them here.

Chandra data are acquired as photon events, with pixel location, arrival time, and the photon energy recorded. The CCD readout time varies between $2.4-3.2 \mathrm{sec}$ for observations of SN 1987A, and the energy resolution on the S3 chip ranges from $\approx 90-150 \mathrm{eV}$ between 1-5 keV. Pixel super-resolution in sky-coordinates is made possible primarily through spacecraft dither (the telescope slews during an observation in a Lissajous pattern with pitch period of $707 \mathrm{sec}$ and yaw period of $1000 \mathrm{sec}$ and an amplitude of 8 arcsec), which allows normal vectors to pixels to be mapped to the sky on average at a resolution of $\approx 0.07$ pix.

Further improvements to photon location are made possible through more precise photon arrival locations. Li et al. (2004) have devised a method called EDSER (Energy Dependent Subpixel Event Resolution) to determine where on the pixel a photon landed based on the shape of the charge collected on the CCD (known as the charge bloom), recorded as the event grade. A fully symmetrical charge bloom centered on a pixel results in the assignment of grade $=0$, and no location correction is applied; for asymmetrical charge blooms, grade $>0$, and an energy dependent correction is applied to shift the photon location from pixel center towards one of the edges. EDSER is automatically applied to ACIS data in Chandra pipeline processing, and has been shown to substantively improve the sharpness of Chandra super-resolved images (see, e.g., http://cxc.harvard.edu/ciao/why/acissubpix.html). Unfortunately, the PSF of the super-resolved EDSER data has not yet been calibrated. As part of the Chandra calibration process, we have constructed an empirical PSF based on real data.

We constructed the empirical PSF by first identifying sources in the Chandra Source Catalog (CSC; http://cxc.harvard.edu/csc) that were flagged as not extended, 

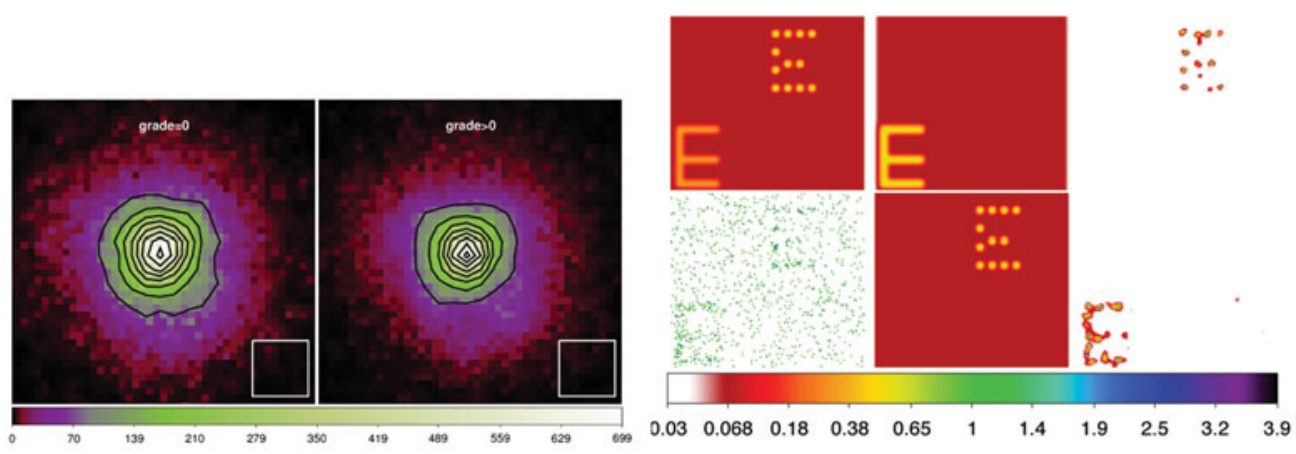

Figure 2. Left set: The ACIS empirical PSF, constructed for grade=0 events (left) and for grade $>0$ events (right). The size of a detector pixel is shown as the square white box.

Right set: Feature detection using LIRA, showing simulation and analysis images. The true image (top left; of size $128 \times 128$ ) consists of two ' $E$ ' shaped sources of similar intensity, one extended and the other spotty, on a flat background and convolved with a Gaussian PSF $(\sigma=6$ bins). A nominal simulated Poisson dataset is shown at bottom left. The images in the second column show baseline models that are input and are assumed to be known a priori: in the top panel, the diffuse "E" is taken to be known, and the unknown point sources are reconstructed; in the bottom panel, the point sources are taken to be known, and the unknown extended source is reconstructed. The images in the third column show the point sources (top) and the extended source (bottom) that are inferred from the multi-scale residuals (Connors et al. 2011).

isolated (with no catalogued sources within $6^{\prime \prime}$ to avoid contamination of the wings), well-observed ( $>200$ counts), on-axis $(<1 \mathrm{arcmin})$, and weak $\left(<0.1 \mathrm{ct} \mathrm{sec}^{-1}\right.$, to avoid pileup). The individual observations were downloaded from the archive in Jan 2017, reprocessed with CIAO v4.9 (see Fruscione et al. 2006) and CALDB v4.5.1.1 (Graessle et al. 2006), derolled so that all the datasets were aligned in the detector frame, and then stacked, resulting in a combined events list of $>90$ kcounts. We then split the accumulated events into separate grade $=0$ and grade $>0$ sets, and constructed images binned at $\frac{1}{8}$ pixel, corresponding to a sky resolution of $0^{\prime \prime} .06125$. The images were then locally adaptively smoothed to reduce statistical fluctuations in the wings. This was achieved by computing smoothed images at a number of smoothing scales, and choosing at each pixel the smallest smoothing scale which achieved a $\mathrm{S} / \mathrm{N}$ of 2 . A background was then estimated from an annulus of $4^{\prime \prime} .4-5^{\prime \prime} .9$ and subtracted out. Pixels that ended up with negative values were filled in by iteratively interpolating from surrounding pixels until no negative values were left. The final PSFs are shown in Figure 2 (left).

A number of future improvements to the PSF are still necessary: first, the current list of sources is not exhaustive, and with the release of version 2 of the CSC (with full RA coverage and newer data included), we expect to nearly double the number of sources included and improve $\mathrm{S} / \mathrm{N}$; second, the current PSF does not distinguish between the back- and front-illuminated ACIS chips; third, we assume that the HETGS does not introduce measurable distortions in the PSF shape, but this assumption has not been verified yet; and fourth, some of the sources selected still have nearby apparently astrophysical structures that are visible to the eye, and though their effect is minimized by derolling and stacking of multiple sources, they must be manually culled in order to obtain a clean PSF profile. Nevertheless, the empirical PSF is a higher fidelity representation of a Chandra/ACIS on-axis source than is achievable currently through raytraces.

\section{LIRA}

We apply the Chandra/ACIS PSF generated as above, weighted by the observed proportions of grade $=0$ and grade $>0$ events, to ACIS observations of SN 1987A to infer the 
true underlying intensity distribution. For this purpose, we use a Bayesian Markov chain Monte Carlo (MCMC) based multi-scale algorithm LIRA (Low-counts Image Reconstruction and Analysis; Esch et al. 2004, Connors \& van Dyk 2007, Connors et al. 2011). LIRA is an open sourced $R$ package, available at https://github.com/astrostat/LIRA.

A given image may be decomposed into a known "baseline" component and an unknown "multi-scale" component. LIRA constructs a Bayesian multi-scale model that is added to the baseline model to fit to the observed image. It can thus be thought of as modeling the residuals, and quantifies the structure in the residuals that exceeds what would be expected under the baseline model. Extended sources are identified in the multi-scale residuals.

We show an example of how LIRA works with two toy examples illustrated in Figure 2 (right). Two artificial sources, both shaped as ' $\mathrm{E}$ ', one extended and one made up of 12 point sources, are analyzed. There are 132 counts in each of the 'E's, and there is a flat background of $\sim 10^{3}$ ct/image. In both cases, we recover the unknown component via multi-scale residuals, which easily pick out the relevant components at high significance. For each case, the 'sky-truth' (convolved with the PSF), the simulated Poisson data, the two different "baseline models", and the LIRA result, with the baseline modeled out are shown. Thus, we can extract the extended ' $E$ ' by adopting the set of 12 point source ' $E$ ' as the null model (top panels), or conversely extract the point sources by adopting the extended 'E' as the null model (bottom panels).

We note that LIRA is not a deconvolution algorithm, but rather draws from the posterior distribution of the intensity at each pixel. A Bayesian reconstruction algorithm has several advantages over regular deconvolution. It removes ambiguity about stopping rules, provides a measure of the uncertainty in the reconstruction via the posterior probability density distribution, and allows the daisy-chaining of analyses such that the result of analyzing one dataset may be easily incorporated into the next. Furthermore, because it is MCMC based, we can obtain error bars on all estimates of interest. The output of this MCMC algorithm is a series of images, one per iteration, describing the multiscale structure in the residuals. The uncertainty in the structures is defined by the distribution of the intensities in these images. For instance, the significance of an extended structure can be determined by comparing this distribution to that derived from images known to be free of structure, i.e., containing only the baseline model (Stein et al. 2015). We have recently used this to detect several jet features in X-rays from high-redshift Quasars (McKeough et al. 2016). Future improvements to the algorithm include accounting for uncertainties in the PSF (mainly based on the techniques pioneered by Lee et al. 2011 and $\mathrm{Xu}$ et al. 2014), and computing the intensities in different passbands without having to run LIRA independently on images made in each band.

\section{SN 1987A Analysis Results}

We have run LIRA on all the ACIS observations available in the Chandra public archive, but present only a subset of representative results here. In all cases, we adopt a flat baseline component that corresponds to the estimated background in the field, and run LIRA for 1000 iterations, and discard the first 400 to account for burn-in. We run LIRA on images in four separate bands: broad $(\mathrm{B} ; 0.5-8 \mathrm{keV})$, soft $(\mathrm{S} ; 0.5-0.9 \mathrm{keV})$, medium $(\mathrm{M} ; 0.9-2 \mathrm{keV})$, and hard $(\mathrm{H} ; 2-8 \mathrm{keV})$. In order to account for the possibility of systematic uncertainties in the PSF, we smooth the images output at each iteration with a Gaussian kernel of $\sigma=\frac{1}{4}$ image pixel. This is an ad hoc correction that disperses $\approx 9 \%$ of the flux in each pixel into the surrounding pixels. We then compute the intensity maps of the X-ray emission from SN 1987A as the smoothed mean of the multi-scale residual 

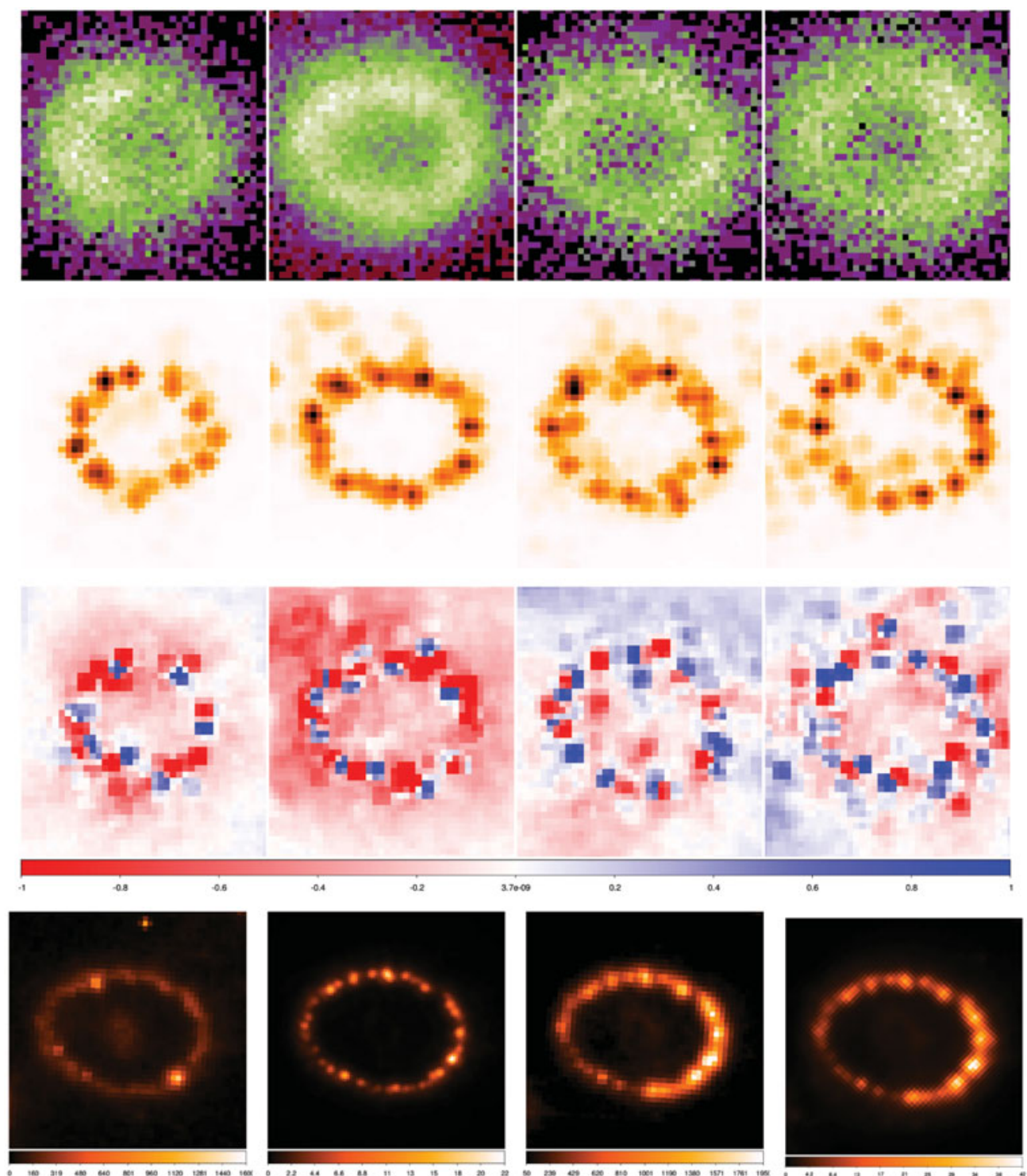

Figure 3. Comparing selected X-ray data and LIRA reconstructions with Hubble. The super-resolved Chandra broad band images are shown in the top row for data from 2000-Dec, 2007-Jul, 2011-Mar, and 2015-Sep. The corresponding LIRA reconstructions are shown in the second row; these images are further Gaussian smoothed to make the structure of the X-ray hot spots visually clearer. The X-ray color $H R_{H S}=\frac{H-S}{H+S}$ maps are showin in the third row, with red and blue shades indicating regions with soft and hard spectra respectively. Contemporaneous Hubble images (from 2000-Nov, 2006-Dec, 2011-Apr, and 2015-May) are shown for comparison in the bottom row (see also Fransson et al. 2015). All plots are $2.4^{\prime \prime} \times 2.4^{\prime \prime}$ in size. Spatial registration across epochs is approximate.

images obtained in each iteration. We also compute the fractional hardness ratios in non-overlapping bands, e.g., $H R_{H S}=\frac{H-S}{H+S}$. We compute the uncertainties on the intensities and on the hardness ratios as the sample standard deviation.

Some results are shown in Figures 3 for a selected set of epochs. Two are for bare ACIS-S observations (ObsIDs 1967 and 7637 from 2000-Dec and 2007-Jul), and two are 
HETGS+ACIS-S observations (ObsIDs 12539 and 16756 from 2011-Mar and 2015-Sep). The X-ray images show a clear increase in the size of the equatorial ring, as well as indications of emission outside the ring, indicating that the shock ejecta is ablating the material of the ring and punching through to the surrounding H II region. Comparison with near-contemporaneous HST images shows a remarkable coincidence of the optical knots with X-ray hot spots. There does not exist a one-to-one correspondence between the optical knots and the X-ray hot spots, but the matches and the morphology cannot be explained as chance coincidences. The ring of X-ray spots is more circular at earlier epochs, suggesting a large separation between the shock front and maximum thermalization, or a large variations in the density of the material in the equatorial ring. This is also borne out by the hardness ratio maps, which shows some X-ray spots dominated by soft emission and others by hard emission. A clear physical interpretation of these features is as yet lacking, and comparisons with hydrodynamic and MHD simulations (e.g., Orlando et al. 2015) are necessary.

In summary, we have developed a method for analyzing the high-resolution X-ray structure in SN 1987A, and have applied it to Chandra ACIS images. The combination of an improved PSF and a powerful reconstruction algorithm has unlocked the potential for significant new understanding in the evolution of the remnant. We plan further improvements to our analysis to use more accurate PSFs, to minimize Monte Carlo error in the reconstructions by running the chains 10x longer, to improve LIRA to better account for uncertainties in the PSF and to analyze the different passbands simultaneously, to evaluate the stability of the reconstructions by analyzing observations made close together, and to determine the statistical significances of X-ray features that have appeared outside the equatorial ring.

\section{Acknowledgements}

We acknowledge support for this work from NASA Contract NAS8-03060 to the Chandra X-ray Center. DvD and AZ acknowledge support from EU Horizon 2020 Project RISE-ASTROSTAT. DvD also acknowledges support from NSF Award number 1513492. We thank Xiao-Li Meng, Nathan Stein, and Salvatore Orlando for useful discussions.

\section{References}

Burrows, D., et al. 1995, ApJ, 452, 6808

Burrows, D., et al. 2000 ApJ, 543, L149

Connors, A. \& van Dyk, D. A. 2007 SCMA IV, ASPC v371, p101

Connors, A., et al. 2011 ADASS XX, v442, p643

Esch, D., et al. 2004 ApJ, 610, 1213

Frank, K., et al. 2016 ApJ, 829, 40

Fransson, C., et al. 2015 ApJL, 806, L19

Fruscione, A., et al. 2006 Proc. SPIE, 6270, 62701V

Graessle, D., et al. 2006 Proc. SPIE, 6270, 62701X

Hasinger, G., et al. 1996 A $E A$ A 312, 9

Helder, et al. 2013 ApJ, 764, 11

Lee, H., et al. 2011 ApJ, 731, 126

$\mathrm{Li}$, et al. 2014 ApJ, 610, 1204

McKeough, K., et al. 2016 ApJ, 833, 123

Orlando, S., et al. 2015 ApJ, 810, 168

Park, T., et al. 2006 ApJ, 646, 1001

Racusin, D., et al. 2009 ApJ, 703, 1752

Stein, N., et al. 2015 ApJ, 813, 66

$\mathrm{Xu}$, J., et al. 2014 ApJ, 794, 97 